\title{
ON THE EULER CHARACTERISTIC OF THE SPACE SATISFYING CONDITION $\left(T^{* *}\right)$
}

\author{
SANG-EON HAN
}

\begin{abstract}
A bstract. In this paper, we prove some results on the Euler characteristic number of a locally nilpotent space and a space satisfying condition $\left(T^{* *}\right)$.
\end{abstract}

\section{Introduction}

The study of the nilpotent space was begun by A. K. Bousfield, P. Hilton, G. Mislin and others $[7,8]$. Especially, the Euler characteristic of the nilpotent space was studied by $R$. H. Lewis [10].

In this paper, we define the condition $\left(T^{*}\right)$ and $\left(T^{* *}\right)$ and the locally nilpotent spaces as the extensive concept of the nilpotent space. Euler characteristic number of the spaces with relation to the conditions $\left(T^{*}\right)$ and $\left(T^{* *}\right)$ will be studied.

Furthermore, we study the homotopy equivalent conditions of the locally nilpotent spaces and spaces satisfying condition $\left(T^{* *}\right)$.

We work in the category of the topological spaces having the homotoppy type of connected $C W$-complexes with base point and denote as the $T$.

\section{Some Properties of the Condition $\left(T^{* *}\right)$}

In this section, we define the locally nilpotent space and condition $\left(T^{*}\right)$ and condition $\left(T^{* *}\right)$ and study their properties respectively.

We recall that locally nilpotent group is the group whose finitely generated subgroups are nilpotent groups [12].

And we denote the category of nilpotent spaces and continuous maps as $T_{N}$.

Now we extend the concept of the nilpotent space like followings.

Definition 2.1. A space $X(\in T)$ is said to be a locally nilpotent space if

(1) $\pi_{1}(X)$ is a locally nilpotent group,

(2) the action $\pi_{1}(X) \times \pi_{n}(X) \rightarrow \pi_{n}(X)$ is nilpotent for all $n \geq 2$ [1].

Received September 17, 1996; revised November 3, 1997.

This work was partially supported by BSRI program, Ministry of Education under the project number BSRI-96-1425 
And we denote the category of locally nilpotent spaces and continuous maps as $T_{L N}$.

We know that the category $T_{N}$ is full subcategory of $T_{L N}$.

Generally, for a group $G$ and a fixed $g \in G$, we denote by $[g, G]$ the subgroup of $G$ generated by all commutators $[g, a]$ which means $g^{-1} a^{-1} g a$ where $a \in G$. Since $[g, a]^{b}=[g, b]^{-1}[g, a b]$ for each $a, b \in G$ (where $\left.a^{b}=b^{-1} a b\right),[g, G]$ is a normal subgroup of $G$.

Definition 2.2. We say that a space $X(\in T)$ satisfies condition $\left(T^{*}\right)$ if for all $g, t \in \pi_{1}(X)$ either $g\left[g, \pi_{1}(X)\right]=t\left[t, \pi_{1}(X)\right]$ or $g\left[g, \pi_{1}(X)\right] \cap t\left[t, \pi_{1}(X)\right]=\phi$.

Now we define the effective concept with respect to the locally nilpotent space.

Definition 2.3. For $X \in T$, we say that $X$ satisfies the condition $\left(T^{* *}\right)$ if for all $g(\neq 1) \in \pi_{1}(X)$, then $g \notin\left[g, \pi_{1}(X)\right]$.

Since the $\left[g, \pi_{1}(X)\right]$ is a normal subgroup of $\pi_{1}(X)$, condition $\left(T^{* *}\right)$ is homotopy invariant property.

In fibration $F \rightarrow E \rightarrow B$, any path $\alpha: I \rightarrow \cdot B$ and singular $q$-complex $g: \triangle^{q} \rightarrow$ $p^{-1}(\alpha(0))$ determine a map $G: \triangle^{q} \times I \rightarrow E$ over $\alpha \circ p r_{2}: \triangle^{q} \times I \rightarrow I \rightarrow B$ and extending $G_{0}=g: \Delta^{q} \times\{0\} \rightarrow E$. if $\alpha$ is a loop, then $G_{1}: \Delta^{q} \times\{1\} \rightarrow E$ is a $q$-simplex in $p^{-1}(\alpha(1))=p^{-1}(\alpha(0))$. Now do elements of $\pi_{1}(B)$ operate on $H_{*}(F)$ [9].

Definition 2.4. A fibration $F \rightarrow E \rightarrow B$ is said to be quasi-hilpotent if the action of $\pi_{1}(B)$ on $H_{*}(F)$ is nilpotent, $* \geq 0$.

Lemma 2.5. For $X \in T_{L N}, X$ satisfies the condition ( $\left.T^{* *}\right)$.

Proof. (Step 1); first, we assert that $X$ satisfies the condition $\left(T^{*}\right)$. Since $\pi_{1}(X)$ is a locally nilpotent group, suppose $c \in a\left[a, \pi_{1}(X)\right] \cap b\left[b, \pi_{1}(X)\right]$ for some $a, b, c \in \pi_{1}(X)$. We only show that $a\left[a, \pi_{1}(X)\right]=b\left[b, \pi_{1}(X)\right]$. If $b \in a\left[a, \pi_{1}(x)\right]$ (for $a, b \in \pi_{1}(X)$ ) then $b\left[b, \pi_{1}(x)\right] \subset a\left[a, \pi_{1}(X)\right]$. We know that

$$
c\left[c, \pi_{1}(X)\right] \subset a\left[a, \pi_{1}(X)\right] \cap b\left[b, \pi_{1}(X)\right]
$$

Clearly, $c=h^{-1} a$ for some $h=\prod_{i=1}^{m}\left[a, g_{i}\right]^{\epsilon_{i}} \in\left[a, \pi_{1}(X)\right]\left(g_{i} \in \pi_{1}(X), \epsilon_{i}= \pm 1\right)$. Let $G_{1}=\left\langle a, g_{1}, \ldots, g_{m}\right\rangle$. Since $a=h c, h \equiv \prod_{i=1}^{m}\left[h, g_{i}\right]^{\epsilon_{i}}$ modulo $\left[c, G_{1}\right]$, that is, $h=$ $\prod_{i=1}^{m}\left[h, g_{i}\right]^{\epsilon_{i}}$ in $\frac{g_{1}}{\left[c, G_{1}\right]}$. However, since the latter group is nilpotent, it follows that $h=1$ in $\frac{G_{1}}{\left[c, G_{1}\right]}$ and $h \in\left[c, G_{1}\right]$. Therefore, $a=h c \in c\left[c, \pi_{1}(X)\right]$ and we get $a\left[a, \pi_{1}(X)\right] \subset$ $c\left[c, \pi_{1}(X)\right][2]$. It follows from $(*)$ that $a\left[a, \pi_{1}(X)\right]=c\left[c, \pi_{1}(X)\right]$.

Similary, $b\left[b, \pi_{1}(X)\right]=c\left[c, \pi_{1}(X)\right]$ and consequently, $a\left[a, \pi_{1}(X)\right]=b\left[b, \pi_{1}(X)\right]$. Thus $X \in T_{L N}, X$ satisfies the condition $\left(T^{*}\right)$.

(Step 2); next, we assert that $X$ above satisfies the condition $\left(T^{* *}\right)$. Assume that $g \in\left[g, \pi_{1}(X)\right]$ for some $(g \neq 1) \in \pi_{1}(X)$. Then $g^{-1} \in\left[g, \pi_{1}(X)\right]$ and $1 \in g\left[g, \pi_{1}(X)\right]$. Thus $g\left[g, \pi_{1}(X)\right] \cap 1\left[1, \pi_{1}(X)\right] \neq \phi$. Since $X$ satisfies the condition $\left(T^{*}\right)$ by (Step 1 ), $g\left[g, \pi_{1}(X)\right]=1$. Since $g \in g\left[g, \pi_{1}(X)\right]$, we have a contradiction.

Remark. If $X(\in T)$ satisfies the condition $\left(T^{*}\right)$, then $X$ satisfies condition $\left(T^{* *}\right)$. But the converse statement need not be true in general [6]. 
Theorem 2.6. For $X \in T_{L N}$, if $b \in\left[a, \pi_{1}(X)\right]$ then $a\left[a, \pi_{1}(X)\right]=b\left[b, \pi_{1}(x)\right]$, for $a, b \in \pi_{1}(X)$.

Proof. If $b \in a\left[a, \pi_{1}(X)\right]$ (for $a, b \in \pi_{1}(X)$ ) then $b\left[b, \pi_{1}(X)\right] \subset a\left[a, \pi_{1}(X)\right]$. Since $X$ satisfies the condition $\left(T^{*}\right)$ by the (Step 1) of Lemma 2.5, thus our proof is completed by the following properties; when $X$ satisfies the condition $\left(T^{*}\right)$, for each $a, b \in \pi_{1}(X)$, if $a\left[a, \pi_{1}(X)\right] \subset b\left[b, \pi_{1}(X)\right]$ then $a\left[a, \pi_{1}(X)\right]=b\left[b, \pi_{1}(X)\right]$. Thus our proof is completed.

\section{Euler Characteristic of the Space Satisfying Condition $\left(T^{* *}\right)$}

In this section, we make results on the Euler characteristic number of the locally nilpotent spaces and spaces satisfying condition $\left(T^{* *}\right)$.

Furthermore, we study about the homotopy equivalent conditions of the nonnilpotent spaces.

Lemma 3.1.[3] Fur finite $X$, if $\pi_{1}(X)$ contains a torsion free normal abelian subgroup $A \neq 1$ which acts nilpotently on $H_{n}(\tilde{X})$ where $n \geq 0$, then $\chi(X)=0$.

Theorem 3.2. For finite $X$ satisfying condition $\left(T^{* *}\right)$, if

(1) the action $\pi_{1}(X) \times H_{n}(\tilde{X}) \rightarrow H_{n}(\tilde{X})$ is nilpotent where $n \geq 0$,

(2) $\pi_{1}(X)(\neq 1)$ is finite,

then $\chi(X)=0$.

Proof. (Step 1); we check the nilpotent property of $\pi_{1}(X)$ under the above hypothesis. So assume that $\pi_{1}(X)$ is not nilpotent, then we don't have finite upper central series of $\pi_{1}(X)$. If $Z_{n}\left(\pi_{1}(X)\right)$ denote the $n$-th center of $\pi_{1}(X)$, we can find an integer $n$ such that $Z_{n+1}\left(\pi_{1}(X)\right)=Z_{n}\left(\pi_{1}(X)\right) \subsetneq \pi_{1}(X)$. It follows that if $x \notin Z_{n}\left(\pi_{1}(X)\right)$, then $\left[x, \pi_{1}(X)\right] \mathbb{E}$ $Z_{n}\left(\pi_{1}(X)\right)$. Choose any $x_{1} \notin Z_{n}\left(\pi_{1}(X)\right)$. we know $\left[x_{1}, \pi_{1}(X)\right] \nsubseteq Z_{n}\left(\pi_{1}(X)\right)$ by above. If $x_{1} \in\left[x_{1}, \pi_{1}(X)\right]$ then we have shown that the condition $\left(T^{* *}\right)$ does not hold, as required, so assume $x_{1} \notin\left[x_{1}, \pi_{1}(X)\right]$. Then choose $x_{2} \in\left[x_{1}, \pi_{1}(X)\right], x_{2} \notin Z_{n}\left(\pi_{1}(X)\right)$. Since $\left[x_{1}, \pi_{1}(X)\right]$ is a normal subgroup of $\pi_{1}(X),\left[x_{2}, \pi_{1}(X)\right] \subseteq\left[x_{1}, \pi_{1}(X)\right]$. If $x_{2} \in\left[x_{2}, \pi_{1}(X)\right]$, we are done.

Otherwise, we have $\left[x_{2}, \pi_{1}(X)\right] \subsetneq\left[x_{1}, \pi_{1}(X)\right]$ but also we noted $\left[x_{2}, \pi_{1}(X)\right] \mathbb{Z}$ $Z_{n}\left(\pi_{1}(X)\right)$. So pick $x_{3} \in\left[x_{2}, \pi_{1}(X)\right], x_{3} \notin Z_{n}\left(\pi_{1}(X)\right)$ and continue. Since $\pi_{1}(X)$ is finite, this process must stop. Afer all we have $\alpha$ for which $x_{\alpha}(\neq 1) \in\left[x_{\alpha}, \pi_{1}(X)\right]$. This is a contradiction to the fact that $X$ satisfies the condition $\left(T^{* *}\right)$. Thus we know that $\pi_{1}(X)$ is nilpotent group.

(Step 2); when $\pi_{1}(X)$ is finite, since $X$ satisfies the condition $\left(T^{* *}\right)$ and by (step $1), \pi_{1}(X)$ is nilpotent group. Thus $X \in T_{N}$. Since $\pi_{1}(X)$ is finite, $\chi(\tilde{X})=\chi(X)$ and another property $\chi(\tilde{X})=\left|\pi_{1}(X)\right| \chi(X)$ where || means the order of $\pi_{1}(X)$ and $\tilde{X}$ means the univeral convering space of $X$ [11]. If $\pi_{1}(X) \neq 1, \chi(X)=0$.

Theorem 3.3. For finitely indexed set $\left\{X_{\alpha}, \alpha \in M:\right.$ finite $\}, X_{\alpha} \in T_{L N}$ for each $\alpha$ with $\pi_{1}\left(X_{\alpha}\right)$ finite then $\chi\left(\prod_{\alpha \in M} X_{\alpha}\right)=0$. 
Proof. (Step 1); we know that $X_{\alpha}$ satisfies the condition $\left(T^{* *}\right)$ by Lemma 2.5. Now let's check the finite product property of condition $\left(T^{* *}\right)$. For set $\left\{X_{\alpha} \mid \alpha \in M\right.$ : finite $\}$, if $X_{\alpha}$ satisfies the condition ( $\left.T^{* *}\right)$ for each $\alpha \in M$ then $\prod_{\alpha \in M} X_{\alpha}$ satisfies the condition $\left(T^{* * *}\right)$ from the following facts; let $G$ be the group $\prod_{\alpha \in M} \pi_{1}\left(X_{\alpha}\right)$ and $P_{\alpha}$ be the projection of $G$ on $\pi_{1}\left(X_{\alpha}\right)$ for any $\alpha \in M$. Suppose that $g \in[g, G]$ for $g \neq 1$ in $G$, there exists $\alpha(\in M)$ such that $P_{\alpha}(g)=g_{\alpha}$ is not identity in $\pi_{1}\left(X_{\alpha}\right)$. Then $g_{\alpha} \in\left[g_{\alpha}, \pi_{1}\left(X_{\alpha}\right)\right]$ this is a contradition to condition $\left(T^{* *}\right)$ (see Lemma 2.5). Thus $\prod_{\alpha \in M} X_{\alpha}$ satisfies the condition $\left(T^{* *}\right)$.

(Step 2); let's check the finite product property of the nilpotent actions. By the nilpotent property of $H_{n}\left(\tilde{X}_{\alpha}\right)$ under the action $\pi_{1}\left(X_{\alpha}\right)$, there is a lower central series of $H_{n}\left(\tilde{X}_{\alpha}\right)$ for each $\alpha \in M$. In this finite product space case, we only prove the arbitrary two product case of $X_{\alpha}, X_{\beta} \in\left\{X_{\alpha}\right\}_{\alpha \in M}$. Put the lower central series of $H_{n}\left(\tilde{X}_{\alpha}\right)$ and $H_{n}\left(\tilde{X}_{\beta}\right)$ under the nilpotent action of $\pi_{1}\left(X_{\alpha}\right)$ and $\pi_{1}\left(X_{\beta}\right)$ respectively like followings; suppose that the nilpotent classes of $X_{\alpha}$ and $X_{\beta}$ are $n$ and $m$ respectively. We get the following:

$$
\begin{aligned}
& H_{n}\left(\tilde{X}_{\alpha}\right) \supset G_{2} \supset G_{3} \supset \cdots \supset G_{j} \supset \cdots \supset G_{n}=\{e\} \\
& H_{n}\left(\tilde{X}_{\beta}\right) \supset E_{2} \supset E_{3} \supset \cdots \supset E_{i} \supset \cdots \supset E_{m}=\{e\}
\end{aligned}
$$

Now we make the following sequence;

$H_{n}\left(\tilde{X}_{\alpha}\right) \times H_{n}\left(\tilde{X}_{\beta}\right) \supset H_{n}\left(\tilde{X}_{\alpha}\right) \times E_{2} \supset G_{2} \times E_{2} \supset \cdots \supset G_{j-1} \times E_{i} \supset G_{j} \times E_{i} \supset$ $G_{j} \times E_{i+1} \supset \cdots \supset G_{n} \times E_{m}=\{e\} \times\{e\} \cdots(*)$.

Then the above sequence (*) is lower central series of $H_{n}\left(\tilde{X}_{\alpha}\right) \times H_{n}\left(\tilde{X}_{\beta}\right)$ under the action $\pi_{1}\left(X_{\alpha}\right) \times \pi_{1}\left(X_{\beta}\right)$ with the componentwise action. Futhermore, the nilpotent class of $X_{\alpha} \times X_{\beta}$ is less than $m \cdot n$. Thus there is a $\pi_{1}\left(\prod_{\alpha \in M} X_{\alpha}\right)$ nilpotent action on $\left.H_{(} \prod_{\alpha \in M} \tilde{X}_{\alpha}\right)$.

Since $\pi_{1}\left(\prod_{\alpha \in M} X_{\alpha}\right)$ is finite, our proof is completed by Theorem 3.2.

Remark. For set $\left\{X_{\alpha} \mid \alpha \in M:\right.$ finite $\}, X_{\alpha} \in T_{L N}$ for each $\alpha \in M$ [5] if and only if $\prod_{\alpha \in M} X_{\alpha} \in T_{L N}[5]$.

Corollary 3.4. For set $\left\{X_{\alpha} \mid \alpha \in M\right.$ : finite $\}, X_{\alpha}\left(\in T_{L N}\right)$ is finite oriented space without boundary then $\chi\left(\prod_{\alpha} \in M X_{\alpha}\right)=0$ if $\pi_{1}\left(X_{\alpha}\right)(\neq 1)$ is finite for any $\alpha \in M$.

Proof. Since $\chi\left(\prod_{\alpha \in M} X_{\alpha}\right)=\prod_{\alpha \in M} \chi\left(X_{\alpha}\right)$, by Theorem 3.2 and Theorem 3.3, our proof is completed.

We recall that a group $G$ satisfies the maximal condition if it has no infinite strictly increasing chain of subgroups [12].

Theorem 3.5. For finite $X\left(\in T_{L N}\right)$, if

(1) $\pi_{1}(X)$ is infinite with the maximal condition on normal subgroups of $\pi_{1}(X)$ or

(2) $\pi_{1}(X)(\neq 1)$ is finite, 
then $\chi(X)=0$.

Proof. (Case 1); when $\pi_{1}(X)$ is finite, we know that $X$ satisfies the condition $\left(T^{* *}\right)$ by the Theorem 2.5. By the similar method of (step 2) of the Theorem 3.2, we get $\chi(X)=0$.

(Case 2); when $\pi_{1}(X)$ is infinite and $\pi_{1}(X)$ has maximal condition on normal subgroups then $\pi_{1}(X)$ is finitely generated nilpotent group. Thus $\pi_{1}(X)$ has the center group of $\pi_{1}(X)$ as the infinite normal abelian subgroup which acts nilpotently on $H_{*}(\tilde{X})$. Then by Lemma 3.1, we have $\chi(X)=0$.

Corollary 3.6. For finite $X$ satisfying condition $\left(T^{* *}\right)$ with $\pi_{1}(X)(\neq 1)$ finite, suppose that

(1) the map $f: \tilde{X} \rightarrow X$ is a universal covering map,

(2) the action $\pi_{1}(X) \times H_{n}(\tilde{X}) \rightarrow H_{n}(\tilde{X})$ is nilpotent for all $n \geq 0$

then $\chi(\tilde{X})=0$.

Proof. Since $\chi(\tilde{X})=\left|\pi_{1}(X)\right| \chi(X)$ where || means the order of $\pi_{1}(X)$ and $\tilde{X}$ means the univeral convering space of $X$, and by the similar proof of the Theorem 3.5, our proof is completed.

In fibration $F_{f} \rightarrow E \stackrel{f}{\rightarrow} B$, if reduced homology group $\tilde{H}_{*}\left(F_{f}\right)=0, * \geq 0$ we call that $f$ is an acyclic map, where $F_{f}$ is a homotopy fiber of $f$.

Corollary 3.7. Let $X\left(\in T_{L N}\right)$ be a finite aspherical polyhedron with $\pi_{1}(X)$ is infinite and has the maximal condition on normal subgroups of $\pi_{1}(X)$ then $\chi(X)=0$.

Proof. See Theorem of S. Rosset [13] and (Step 2) of Theorem 3.5.

Theorem 3.8. For finite $X$ satisfying condition $\left(T^{* *}\right)$, if

(1) the action $\pi_{1}(X) \times H_{n}(\tilde{X}) \rightarrow H_{n}(\tilde{X})$ is nilpotent for all $n \geq 0$,

(2) $f: X \rightarrow Y$ is an acyclic map with $\pi_{1}(X)$ finite,

then $\chi(Y)=0$.

Proof. By the (step 1) of the Theorem 3.2, $\pi_{1}(X)$ is a nilpotent group. Thus $X \in T_{N}$. From the fact that $f: X \rightarrow Y$ is an acyclic map and the classical homotopy exact sequence of fibration: $F_{f} \rightarrow X \stackrel{f}{\rightarrow} Y$, we know that $\pi_{1}(f)$ is an epimorphism, because $\pi_{0}\left(F_{f}\right)=0$. Furthermore $H_{1}\left(F_{f}\right) \cong \frac{\pi_{1}\left(F_{f}\right)}{\left[\pi_{1}\left(F_{f}\right), \pi_{1}\left(F_{f}\right)\right]}=0$ where [,] means the commutator subgroup. $\pi_{1}\left(F_{f}\right)$ is a perfect group and the homomorphic image of a perfect group is also a perfect group. Thus $\pi_{1}(X) \cong \frac{\pi_{1}(Y)}{P \pi_{1}(X)}$ where $P \pi_{1}(X)$ means a perfect normal subgroup of $\pi_{1}(X)$. Since $X \in T_{N}, \chi(X)=0$ under the above condition and $P \pi_{1}(X)$ is trivial. Thus $\pi_{1}(f)$ is an isomorphism. By use of the Hurewicz Theorem inductively, $\pi_{i}\left(F_{f}\right)=0$. Thus $f$ is a weak homotopy equivalence. By the Whitehead Theorem [4], $f$ is a homotopy equivalence. Therefore, our proof is completed.

Theorem 3.9. For finite $X$ satisfying condition $\left(T^{* *}\right)$, if 
(1) $f: X \rightarrow Y$ is quasi-nilpotent homology equivalence with $\pi_{1}(X)$ finite,

(2) the action $\pi_{1}(X) \times H_{n}(\tilde{X}) \rightarrow H_{n}(\tilde{X})$ is nilpotent for all $n \geq 0$

Then $\chi(Y)=0$.

Proof. By the (step 1 ) of the Theorem 3.2, $X$ is a nilpotent space. We know the homotopy fiber $F_{f}$ of $f$ is also nilpotent space. From the fact that $f$ is quasi-nilpotent, $Y$ is a nilpotent space. Thus we conclude that $f$ is a nilpotent map. Since $f$ is nilpotent map and homology equivalence, $f$ is a homotopy equivalence. Thus our proof is completed.

Corollary 3.10. For finite $X\left(\in T_{L N}\right)$ if $f: X \rightarrow Y$ is quasi-nilpotent homology equivalence with $\pi_{1}(X)$ finite, then $\chi(Y)=0$.

Proof. By Lemma 2.5 and Theorem 3.9, our proof is completed.

\section{References}

[1] A. K. Bousfield and D. M. Kan, "Homotopy Limits," Completions and Localization, L. N. S. vol. 304, Springer-Verlag, Berlin, 1972.

[2] M. Dokuchaev, "On the property of nilpotent groups," Canad. Math. Bull. 37(2)(1994), 174-177.

[3] B. Eckmann, "Nilpotent group action and Euler characteristic," L. N. M. vol. 1298, Springer-Verlag, New York, 1985.

[4] B. Gray, "Homotopy theory," Academic press, New York, 1975,

[5] S. E. Han, "Some properties of the spaces stisfying condition $\left(T^{* *}\right)$," (preprint) (1996).

[6] __ " $\left(T^{* *}\right)$-properties of the residually nilpotent spaces," (preprint) (1996).

[7] P. Hilton, "Nilpotent action on nilpotent groups," Proc. Aust. Summer Institute, (1974).

[8] P. Hilton, G. Mislin and J. Roitberg, "Localization of Nilpotent Groups and Spaces," 1975.

[9] — and J. Roiterberg, "On the Zeeman comparison theorem for the homology of quasinilpotent fibrations," Quart. J. Math., Oxford 27(1976), 433-444.

[10] R. H. Lewis, "Homology and cell structure of nilpotent space," Trans. of the A. M. S., $290(2)(1985), 747-760$.

[11] G. Mislin, "Finitely dominated nilpotent space," Annal of Math., 103(1976), 547-556.

[12] D. J. S. Robinson, "A Courses in the Theory of Groups," Springer-Verlag, New York, 1980.

[13] S. Rosset, "A vanishing theorem for Euler characteristics."

Department of Mathematics, College of Natural Sciences, Honam University, Kwangju 506-090, Korea. 